Physics Vol. 3, No. 1, pp. 47-67, 1967. Physics Publishing Co. Printed in Great Britain.

\title{
A SIMPLE DISCUSSION OF su(3) AND su(6)
}

\author{
GÖRAN FÄLDT \\ Department of Theoretical Physics, University \\ of Lund, Lund, Sweden \\ (Received 5 June 1966)
}

\begin{abstract}
This note is intended to give an elementary derivation of some basic properties of the unitary groups $S U(3)$ and $S U(6)$ used in current particle physics. It contains no new results and is intended as an introduction for the non-specialist.

The success of $S U(3)$ has stimulated the search for higher symmetries in particle physics. In these investigations one sometimes refers to rather deep theorems of group theory. Furthermore, many results are published in the form of short letters. In consequence, the new ideas and techniques are often difficult to understand for physicists not familiar with all the technicalities of group theory. Nevertheless, many results can be obtained by straight forward generalizations of methods encountered in the elementary theory of angular momentum.

Here we shall confine ourselves to the derivation of the representations of $S U(3)$ and $S U(6)$ and the grouping of particles into multiplets. Mass-formulas and magnetic moments, as well as many other applications, can be worked out with the same method but here we only give the background and leave the detailed applications to the interested reader.
\end{abstract}

We start with a review of the group $S U(2)$ with special attention to properties independent of dimensions.

\section{Discussion of $S U(2)$}

THE group $S U(2)$ can conveniently be defined as follows. Consider the space $C_{2}$ of ordered pairs of complex numbers. We then define $S U(2)$ as the group of all linear transformations $a$ on $C_{2}$ with the properties

$$
\begin{aligned}
& a a^{*}=a^{*} a=1 \\
& \operatorname{det} a=1
\end{aligned}
$$

In $C_{2}$ we introduce the basis vectors

$$
\xi_{1}=\left(\begin{array}{l}
1 \\
0
\end{array}\right), \quad \xi_{2}=\left(\begin{array}{l}
0 \\
1
\end{array}\right) .
$$


With this basis the unitary $2 \times 2$ matrices with determinant one form a representation of the elements of $S U(2)$. Every matrix of this kind can be written

$$
a=e^{i F} \text {. }
$$

As $a$ is unitary, $F$ is hermitian. From

$$
\operatorname{det} a=e^{i} S_{p}[F]
$$

we find $S p[F]=0$. But we have only three linearly independent traceless $2 \times 2$ matrices. In the theory of angular momentum these are usually chosen as $\sigma_{x}, \sigma_{y}$ and $\sigma_{z}$. Here we will make another choice

$$
\begin{aligned}
& M_{11}=\left(\begin{array}{ll}
1 & 0 \\
0 & 0
\end{array}\right)-\frac{1}{2}\left(\begin{array}{ll}
1 & 0 \\
0 & 1
\end{array}\right)=\frac{1}{2} \sigma_{z}, \\
& M_{22}=\left(\begin{array}{ll}
0 & 0 \\
0 & 1
\end{array}\right)-\frac{1}{2}\left(\begin{array}{ll}
1 & 0 \\
0 & 1
\end{array}\right)=-\frac{1}{2} \sigma_{z} \\
& M_{12}=\left(\begin{array}{ll}
n & 1 \\
0 & 0
\end{array}\right)=\frac{1}{2} \sigma_{+}=\frac{1}{2}\left(\sigma_{x}+i \sigma_{y}\right), \\
& M_{21}=\left(\begin{array}{ll}
0 & 0 \\
1 & 0
\end{array}\right)=\frac{1}{2} \sigma_{-}=\frac{1}{2}\left(\begin{array}{ll}
\sigma_{x}-i & \left.\sigma_{y}\right) .
\end{array}\right.
\end{aligned}
$$

The matrices $M_{k l}$ are not linearly independent but satisfy

$$
M_{11}+M_{22}=\sum_{k} M_{k k}=0 \text {. }
$$

Furthermore $M_{k l}^{*}=M_{l k}$. In terms of these four matrices

$$
F=\sum_{k, l} \alpha_{k l} M_{k l} .
$$

As $F$ is hermitian, the numbers $\alpha_{k l}$, (which are not unique because of the linear dependence of the matrices $M_{k l}$ ) may be taken to satisfy

$$
\alpha_{k l}^{*}=\alpha_{l k} .
$$

The matrices $M_{k l}$ are the matrices for the generators of the group $S U(2)$ in the 2-dimensional representation. They satisfy 


$$
\left[M_{k l}, M_{r s}\right]=\delta_{l r} M_{k s}-\delta_{k s} M_{r l}
$$

The non-diagonal matrices have the property

$$
M_{k l} \xi_{m}=\delta_{l m} \xi_{k}, \quad k \neq l
$$

while the vectors $\xi_{m}$ are eigenvectors of the diagonal matrices $M_{k k}$.

The space spanned by $\xi_{1}$ and $\xi_{2}$ is denoted $\square$ and corresponds to spin $\frac{1}{2}$. From the space we can construct bases for new representations of $S U(2)$ by adding spins. Let us start with the addition of two spins $\frac{1}{2}$. We then form a new vector space $\square \times \square$ whose basis is the formal products $\xi_{i} \eta_{j}$, where $\xi_{i}$ and $\eta_{j}$ are basis vectors in two different spaces $\square$. The space $\square \times \square$ is 4-dimensional and the generators are

$$
M_{k l}=M_{k l} \cdot 1+1 \cdot M_{k l} \text {. }
$$

Here the operators standing to the left of the dots operate in the space spanned by the $\xi_{i}$ and the operators standing to the right operate in the space spanned by the $\eta_{i}$. This is just the usual way of adding two commuting spin operators $\bar{s}_{1}+\bar{s}_{2}=\bar{s}$. The $M_{k l}$ satisfy

$$
\left[\mathbf{M}_{k l}, \mathbf{M}_{r s}\right]=\delta_{l r} \mathbf{M}_{k s}-\delta_{k s} \mathbf{M}_{r l} \text {. }
$$

We want to decompose $\square \times \square$ into subspaces corresponding to definite spin, i.e. spaces that are invariant and irreducible under $S U(2)$. A space is said to be invariant if every transformation of $S U(2)$ operating on vectors in the space gives new vectors in the same space. It is irreducible if from any given vector in the space we can reach any other vector of the same space by the application of transformations of $S U(2)$. In view of (3) and (7) it is sufficient to prove the invariance for the operators $M_{k l}$. In the same way the space is irreducible if from any given basis vector of the space we can reach any other basis vector by repeated application of operators $M_{k l}$.

In our special case we know that we have two invariant and irreducible subspaces corresponding to spin 1 and spin 0 . The space corresponding to spin 1 is denoted $\square$ and has basis vectors

$$
S_{11}=\xi_{1} \eta_{1}, \quad S_{12}=\frac{1}{2}\left[\xi_{1} \eta_{2}+\xi_{2} \eta_{1}\right], \quad S_{22}=\xi_{2} \eta_{2} .
$$

The space corresponding to spin 0 is denoted $\boxminus$ and has the single basis vector

$$
A_{12}=\frac{1}{2}\left[\xi_{1} \eta_{2}-\xi_{2} \eta_{1}\right] \text {. }
$$

We note that $S_{12}$ and $A_{12}$ are not normalized. This is so because we want to avoid unnecessary complications of normalization factors in later formulae.

The space $\square$ is irreducible and invariant. This follows from the relations

$$
\begin{array}{lll}
\mathbf{M}_{21} S_{11}=2 S_{12} & \mathbf{M}_{21} S_{12}=S_{22} & \mathbf{M}_{21} S_{22}=0 \\
\mathbf{M}_{12} S_{11}=0 & \mathbf{M}_{12} S_{12}=S_{11} & \mathbf{M}_{12} S_{22}=2 S_{12} .
\end{array}
$$


In the space $\square$ we have

$$
M_{12} A_{12}=M_{21} A_{12}=0
$$

We need not bother about the diagonal operators as $\xi_{i}$ and $\eta_{j}$ are eigenvectors of these operators and the vectors $\xi_{i} \eta_{j}$ and $\xi_{j} \eta_{i}$ obviously have the same eigenvalues. The basis vectors given above are thus eigenvectors of $M_{11}$ and $M_{22}$.

Summarizing we can write

$$
\begin{aligned}
& S_{i j}=\frac{1}{2}\left[\xi_{i} \eta_{j}+\xi_{j} \eta_{i}\right] . \\
& A_{i j}=\frac{1}{2}\left[\xi_{i} \eta_{j}-\xi_{j} \eta_{i}\right] .
\end{aligned}
$$

We see that $S_{i j}$ is symmetric in the indices $i, j$ and that $A_{i j}$ is antisymetric in the indices $i, j$. That the spaces $\square$ and $\boxminus$ span the space $\square \times \square$ can be seen from a simple counting of dimensions but also from the identity

$$
\xi_{i} \eta_{j} \equiv S_{i j}+A_{i j}
$$

We use the formal expression

$$
x \square=\square+E
$$

to signify that the two invariant and irreducible spaces $\square$ and $\boxminus$ together span the space $\square \times \square$.

Let us now add three spins $\frac{1}{2}$. We then form the vector space $\square \times \square \times \square$ whose basis vectors are the products $\xi_{i} \eta_{j} \zeta_{k}$. The generators are now

$$
M_{k l}=M_{k l} \cdot 1 \cdot 1+1 \cdot M_{k l} \cdot 1+1 \cdot 1 \cdot M_{k l},
$$

and the commutation relations are the same as those of $M_{k l}$. We know that $\square \times \square \times \square$ can be decomposed into invariant subspaces corresponding to spin $\frac{3}{2}, \frac{1}{2}$ and $\frac{1}{2}$.

Let us start with a discussion of the space $\square$ corresponding to spin $\frac{3}{2}$. This space contains the vector $S_{11.1}=\xi_{1} \eta_{1} \zeta_{1}$. The other vectors are obtained in a well-known way

$$
\begin{aligned}
& M_{21} S_{111}=\xi_{1} \eta_{1} \zeta_{2}+\xi_{1} \eta_{2} \zeta_{1}+\xi_{2} \eta_{1} \zeta_{1}=3 S_{112}, \\
& M_{21} S_{112}=\frac{2}{3}\left[\xi_{1} \eta_{2} \zeta_{2}+\xi_{2} \eta_{1} \zeta_{2}+\xi_{2} \eta_{2} \zeta_{1}\right]=2 S_{122}, \\
& M_{21} S_{122}=\xi_{2} \eta_{2} \zeta_{2}=S_{222}, \\
& M_{21} S_{222}=0 .
\end{aligned}
$$


The operators $M_{12}$ work in an analogous way and the vectors $S_{i j k}$ are eigenvectors of $M_{11}$ and $M_{22}$. Thus the space $\square \amalg$ spanned by these four vectors is invariant and irreducible.

Summarizing we can write

$$
S_{i j k}=\frac{1}{6}\left[\xi_{i} \eta_{j} \zeta_{k}+\xi_{i} \eta_{k} \zeta_{j}+\xi_{j} \eta_{i} \zeta_{k}+\xi_{j} \eta_{k} \zeta_{i}+\xi_{k} \eta_{j} \zeta_{i}+\xi_{k} \eta_{i} \zeta_{j}\right] .
$$

and $S_{i j k}$ is symmetric under any permutation of the indices $i, j, k$.

We now turn our attention to the 2-dimensional subspaces of spin $\frac{1}{2}$. States of spin $\frac{1}{2}$ are, e.g. obtained by first coupling two spins to a resulting spin zero and then adding the remaining spin. This can be done in three different ways

$$
\begin{aligned}
& {\left[\xi_{1} \eta_{2}-\xi_{2} \eta_{1}\right] \zeta_{i},} \\
& {\left[\xi_{1} \zeta_{2}-\xi_{2} \zeta_{1}\right] \eta_{i} .} \\
& {\left[\eta_{1} \zeta_{2}-\eta_{2} \zeta_{1}\right] \xi_{i} .}
\end{aligned}
$$

These combinations are not linearly independent. This is related to the fact that we have only two independent spaces of spin $\frac{1}{2}$. We choose the following independent combinations

$$
\begin{aligned}
& F_{1 i 2}=\frac{1}{3}\left\{\left[\xi_{1} \zeta_{2}-\xi_{2} \zeta_{1}\right] \eta_{i}+\left[\eta_{1} \zeta_{2}-\eta_{2} \zeta_{1}\right] \xi_{i}\right\} . \\
& F_{12 i}=\frac{1}{3}\left\{\left[\xi_{1} \eta_{2}-\xi_{2} \eta_{1}\right] \zeta_{i}+\left[\eta_{2} \zeta_{1}-\eta_{1} \zeta_{2}\right] \xi_{i}\right\} .
\end{aligned}
$$

The basis vectors $F_{12}$ and $F_{12}^{\prime}$ are of the same structure and we use the symbol $\square$ to denote a space spanned by $F_{1 i 2}$ or $F_{12}$. Because of the similarity between these spaces we only discuss the one spanned by $F_{1 i 2}$. It is invariant and irreducible as

$$
\begin{array}{ll}
M_{21} F_{112}=F_{122} & M_{12} F_{112}=0 \\
M_{21} F_{122}=0 & M_{12} F_{122}=F_{112} .
\end{array}
$$

As before, the vectors $F_{1 i 2}$ are eigenvectors of $M_{11}$ and $M_{22}$.

We generally define

$$
\begin{aligned}
& F_{i j k}=\frac{1}{3}\left\{\left[\xi_{i} \zeta_{k}-\xi_{k} \zeta_{i}\right] \eta_{j}+\left[\eta_{i} \zeta_{k}-\eta_{k} \zeta_{i}\right] \xi_{j}\right\} . \\
& F_{i j k}^{\prime}=\frac{1}{3}\left\{\left[\xi_{i} \eta_{j}-\xi_{j} \eta_{i}\right] \zeta_{k}+\left[\eta_{j} \zeta_{i}-\eta_{i} \zeta_{j}\right] \xi_{k}\right\} .
\end{aligned}
$$

That the space $\square \square$ and the two spaces $\square$ together span the space $\square \times \square \times \square$ can be seen by counting dimensions or from the identity 


$$
\xi_{i} \eta_{j} \zeta_{k} \equiv S_{i j k}+F_{i j k}+F_{i j k}^{\prime}
$$

We formally describe this property by

$$
\square \times \square \times \square=\square+\square+\square \text {. }
$$

\section{Discussion of $S U(n)$}

We now want to generalize our results for $S U(2)$ to $S U(n)$. The underlying vector space is then $C_{n}$, the space of ordered $n$-tuples of complex numbers. Those linear transformations $a$ on $C_{n}$ with properties

$$
\begin{aligned}
& a a^{*}=a^{*} a=1, \\
& \operatorname{det} a=1
\end{aligned}
$$

constitute the group $S U(n)$. If we introduce the basis

$$
\xi_{1}=\left(\begin{array}{c}
1 \\
0 \\
0 \\
\cdot \\
\cdot \\
0
\end{array}\right) \quad \xi_{2}=\left(\begin{array}{c}
0 \\
1 \\
0 \\
\cdot \\
\cdot \\
\cdot \\
0
\end{array}\right) \ldots \ldots \quad \xi_{n}=\left(\begin{array}{c}
0 \\
0 \\
\cdot \\
\cdot \\
\cdot \\
0 \\
1
\end{array}\right)
$$

in $C_{n}$, the unitary $n \times n$ matrices with determinant one form a representation of the elements of the group. We write them in the form

$$
a=e^{i F}
$$

with hermitian $F$ and find as before the condition $S_{p}[F]=0$. We further introduce the traceless $n \times n$ matrices $M_{k l}, k, l=1, \ldots, n$ defined by

$$
\begin{aligned}
& \left(M_{k l}\right)_{\alpha \beta}=\delta_{k \alpha} \delta_{l \beta}, \quad k \neq l . \\
& \left(M_{k k}\right)_{\alpha \beta}=\delta_{k \alpha} \delta_{k \beta}-\frac{1}{n} \delta_{\alpha \beta} .
\end{aligned}
$$

Only $n-1$ of the diagonal matrices are independent as

$$
\sum_{k} M_{k k}=0 \text {. }
$$

The following relations are simple generalizations of those found for $S U(2)$ 


$$
\begin{aligned}
& F=\sum \alpha_{k l} M_{k l}, \quad \alpha_{k l}^{*}=\alpha_{l k}, \\
& {\left[M_{k l}, M_{r s}\right]=\delta_{l r} M_{k s}-\delta_{k s} M_{r l},} \\
& M_{k l} \xi_{m}=\delta_{l m} \xi_{k}, \quad k \neq l, \\
& M_{k k} \xi_{m}=\left(\delta_{k m}-\frac{1}{n}\right) \xi_{m} .
\end{aligned}
$$

In conjunction with our previous language we use the symbol $\square$ to denote the space spanned by $\xi_{i}, i=1, \ldots, n$. It is a basis for a representation of $S U(n)$. Other representations can be obtained by studying product spaces. Let us start with a product of two spaces denoted $\square \times \square$ and spanned by $\xi_{i} \eta_{j}, i, j=1, \ldots, n$. In this space the operators $M_{k l}$ are defined as for $S U(2)$. We now want to decompose $\square \times \square$ into invariant and irreducible subspaces. Generalizing our result for $S U(2)$ we guess that the spaces spanned by

$$
\begin{aligned}
& S_{i j}=\frac{1}{2}\left[\xi_{i} \eta_{j}+\xi_{j} \eta_{i}\right], \\
& A_{i j}=\frac{1}{2}\left[\xi_{i} \eta_{j}-\xi_{j} \eta_{i}\right]
\end{aligned}
$$

are invariant and irreducible.

The vectors $S_{i j}$ span the space $\square$. As $S_{i j}$ is symmetric in $i, j$ the linearly independent vectors are obtained if we confine ouselves to indices $i \leqslant j$. The space $\square$ is invariant as every vector $S_{i j}$ is an eigenvector of $\mathbf{M}_{\alpha \alpha}$, and for $\mathbf{M}_{\alpha \beta}, \alpha \neq \beta$,

$$
\mathbf{M}_{\alpha \beta} S_{i j}=\delta_{i \beta} S_{j \alpha}+\delta_{j \beta} S_{i \alpha} \text {. }
$$

The irreducibility can be seen as follows. Every $S_{i j}$ can be transformed to any $S_{\alpha \beta}$. If only one index needs to be changed

$$
M_{\alpha i} S_{i \beta}=\left(1+\delta_{i \beta}\right) S_{\alpha \beta}, \quad \alpha \neq i
$$

If both indices must be changed this is achieved by

$$
\mathbf{M}_{\alpha i} \mathbf{M}_{\beta j} S_{i j}=\left(1+\delta_{i \beta}+\delta_{i j}+\delta_{i j} \delta_{\beta i}\right) S_{\alpha \beta}, \quad \alpha \neq i, \beta \neq j .
$$

This proves the irreducibility.

The space spanned by the vectors $A_{i j}$ is denoted $\boxminus$. As $A_{i j}$ is antisymmetric under a permutation of indices, the linearly independent nonzero vectors are obtained for $i<j$. That

is invariant is seen as for $\square$. The irreducibility is also seen in an analogous way. Every $A_{i j}, i \neq j$, can be transformed to any $A_{\alpha \beta}, \alpha \neq \beta$. If one index is correct 


$$
\mathbf{M}_{\alpha i} A_{i \beta}=A_{\alpha \beta}, \quad i \neq \alpha
$$

If they have no index in common

$$
\mathbf{M}_{\alpha i} \mathbf{M}_{\beta j} A_{i j}=A_{\alpha \beta}, \quad i \neq \alpha, \beta, \quad j \neq \alpha, \beta .
$$

Let us now calculate the dimensions of these spaces. There are $\frac{1}{2} n(n+1)$ possibilities of choosing indices $i \leqslant j \leqslant n$. Thus $\operatorname{dim}(\square)=\frac{1}{2} n(n+1)$. Further, there are $\frac{1}{2} n(n-1)$ possibilities of choosing indices $i<j \leqslant n$ and, consequently, $\operatorname{dim}(\theta)=\frac{1}{2} n(n-1)$. As $\operatorname{dim}(\square)+\operatorname{dim}(\theta)=n^{2}$ and the spaces are disjoint, they together span $\square \times \square$. This can also be seen from the identity

$$
\xi_{i} \eta_{j} \equiv S_{i j}+A_{i j}
$$

Also for general $n$ we can therefore write

$$
\square \times \square=\square+B \text {. }
$$

Encouraged by this success we turn to the space $\square \times \square \times \square$ spanned by the vectors $\xi_{i} \eta_{j} \zeta_{k}, i, j, k=1, \ldots, n$. The generators $M_{k l}$ are defined as for $n=2$ and satisfy the commutation relations (9). We expect that the spaces spanned by the vectors

$$
\begin{aligned}
& S_{i j k}=\frac{1}{6}\left[\xi_{i} \eta_{j} \zeta_{k}+\xi_{i} \eta_{k} \zeta_{j}+\xi_{j} \eta_{i} \zeta_{k}+\xi_{j} \eta_{k} \zeta_{i}+\xi_{k} \eta_{i} \zeta_{j}+\xi_{k} \eta_{j} \zeta_{i}\right], \\
& F_{i j k}=\frac{1}{3}\left\{\left[\xi_{i} \zeta_{k}-\xi_{k} \zeta_{i}\right]_{\eta_{j}}+\left[\eta_{i} \zeta_{k}-\eta_{k} \zeta_{i}\right] \xi_{j}\right\} \\
& F_{i j k}^{\prime}=\frac{1}{3}\left\{\left[\xi_{i} \eta_{j}-\xi_{j} \eta_{i}\right] \zeta_{k}+\left[\eta_{j} \zeta_{i}-\eta_{i} \zeta_{j}\right] \xi_{k}\right\} .
\end{aligned}
$$

are each invariant and irreducible. That the different spaces are invariant is trivial and need only be discussed for $S_{i j k}$. Every term in $S_{i j k}$ is an eigenvector of $M \alpha \alpha$ and since we have the same index combinations in each of them they all have the same eigenvalue. Consequently, $S_{i j k}$ is an eigenvector of every $M_{\alpha \alpha}$. For the non-diagonal $M_{\alpha \beta}$

$$
M_{\alpha \beta} S_{i j k}=\delta_{\beta i} S_{\alpha j k}+\delta_{\beta j} S_{i \alpha k}+\delta_{\beta k} S_{i j \alpha} \text {. }
$$

proving the invariance.

We start with a discussion of the space spanned by the vectors $S_{i j k}$ and denoted $\square$. Every $S_{i j k}$ of this space can be transformed to every $S_{\alpha \beta \gamma}$. If they have no index in common

$$
\begin{aligned}
& \mathbf{M}_{\alpha i} M_{\beta j} M_{\gamma k} S_{i j k}=\left(1+\delta_{i k}+\delta_{j k}\right)\left(1+\delta_{i j}+\delta_{j \gamma}\right) \\
& \times\left(1+\delta_{i \beta}+\delta_{i \gamma}\right) S_{\alpha \beta \gamma}, \quad i \neq \alpha, \beta, \gamma \text { etc. }
\end{aligned}
$$


If some indices are correct the reduction is even simpler and we omit the formulae. This proves the irreducibility.

We then determine the dimension of $\square \longrightarrow$. As $S_{i j k}$ is symmetric in all three indices only the index combinations $i \leqslant j \leqslant k$ give linearly independent vectors. As this is equivalent to $i<j+1<k+2$ we find

$$
\operatorname{dim}(\square \square)=\left(\begin{array}{c}
n+2 \\
3
\end{array}\right)=\frac{1}{6}(n+2)(n+1) n .
$$

We now turn to the space

spanned by the vectors $F_{i j k}$. Before proceeding we determine the linearly independent vectors. As $F_{i j k}=-F_{k j i}$ we can confine ourselves to indices $i<k$. Another restriction is $i \leqslant j$. This is not so obvious but follows from the identity

$$
F_{i j k}=F_{j i k}-F_{j k i} \text {. }
$$

In $F_{i j k}$ we can always arrange so that $i<k$. When this condition is fulfilled we see from (54) that if $j<i$, both vectors on the right hand side have indices in correct order. The different ways of choosing $i \leqslant j \leqslant n, i<k \leqslant n$ give independent vectors and

$$
\operatorname{dim}(\boxminus)=\frac{1}{3} n\left(n^{2}-1\right) \text {. }
$$

The irreducibility is proven in two steps. We have already discussed $n=2$ and we now discuss $n>2$. Given any vector $F_{i j k}, i \leqslant j, i<k$ we can reduce it to $F_{112}$. If $i>1$ we have

$$
\mathbf{M}_{2 k} \mathbf{M}_{1 j} \mathbf{M}_{1 i} F_{i j k}=\left(1+\delta_{i j}\right) F_{112} \text {. }
$$

If some indices are already correct, a smaller number of operations will suffice. From $F_{112}$ any $F_{i j k}$ can be constructed. Using (54) we find

$$
\begin{aligned}
& M_{31} F_{112}=2 F_{132}-F_{123}, \\
& M_{21} M_{32} F_{112}=2 F_{123}-F_{132} .
\end{aligned}
$$

giving the two vectors $F_{123}$ and $F_{132}$. From these two any $F_{i j k}, i \leqslant j, i<k$ can be onstructed. If $j=1$ also $i=1$ and we get $F_{i j k}=M_{k 3} M_{j 2} F_{123}$. If $j=2$ or 3 we choose the approriate $F$ constructed from $F_{112}$. When $j=2$ we thus start from $F_{123}$ and get $F_{i j k}=M_{i 1} M_{k 3} F_{123}$. Finally when $j>3$ we find $F_{i j k}=M_{i 1} M_{k 3} M_{j 2} F_{123}$. Thus the irreducibility is proved.

Do the space $\square \square$ and the two spaces $\square$ together span the space $\square \times \square \times \square$ ? Their total dimension is $\frac{1}{6}(n+2)(n+1) n+2 \cdot \frac{1}{3} n\left(n^{2}-1\right)=n^{3}-\frac{1}{6}(n-2)(n-1) n$. Consequently we have at least one more interesting subspace. Which one? The vectors $S_{i j k}$ are symmetric in all indices and the vectors $F_{i j k}$ are antisymmetric in two indices. But concerning symmetry we can also think of vectors that are antisymmetric under change of any two indices. Such a combination is

$$
A_{i j k}=\frac{1}{h}\left[\xi_{i} \eta_{j} \zeta_{k}-\xi_{i} \eta_{k} \zeta_{j}-\xi_{j} \eta_{i} \zeta_{k}+\xi_{j} \eta_{k} \zeta_{i}+\xi_{k} \eta_{i} \zeta_{j}-\xi_{k} \eta_{j} \zeta_{i}\right] .
$$

which span a space denoted $\theta$. Because of the symmetry only those vectors $A_{i j k}$ with 
$i<j<k$ are linearly independent and different from zero. It is clear that this condition cannot be fulfilled for $n=2$. The space is obviously invariant. It is also irreducible. Any $A_{i j k}, 1<i<j<k$, can be reduced to $A_{123}$ by

$$
\mathbf{M}_{3 k} \mathbf{M}_{2 j} \mathbf{M}_{1 i} A_{i j k}=A_{123} \text {. }
$$

If some indices are correct, a smaller number of steps will suffice. On the other hand, any $A_{i j k}, 1<i<j<k$ can be obtained from $A_{123}$ as

$$
A_{i j k}=\mathbf{M}_{i 1} \mathbf{M}_{j 2} \mathbf{M}_{k 3} A_{123} \text {. }
$$

Also here a smaller number of steps will suffice if some indices are correct. As $1 \leqslant i<j<$ $k \leqslant n$ we find

$$
\operatorname{dim}(\theta)=\left(\begin{array}{l}
n \\
3
\end{array}\right)=\frac{1}{6} n(n-1)(n-2) .
$$

From the identity

$$
\xi_{i} \eta_{j} \zeta_{k} \equiv S_{i j k}+A_{i j k}+F_{i j k}+F_{i j k}^{\prime}
$$

it follows that our four disjoint spaces together span $\square \times \square \times \square$ and we write

$$
x \square \times \square=\square+\theta+\boxminus+\theta \text {. }
$$

\section{Conjugate Representations}

A representation is not always determined by its dimension. Thus we have two $n$-dimensional representations. One of these was used in the definition of $S U(n)$. We will now consider the other one. Here the underlying vector space is a subspace in the space obtained by forming formal products of $n-1$ spaces $C_{n}$. This space is spanned by the basis vectors

$\prod_{i=1}^{n-1} \xi_{l_{i}}^{(i)}$, where the indices $l_{i}$ independently run from 1 to $n$. From these basis vectors we form those linear combinations that are antisymmetric under a change of any two of the $n-1$ indices. They are

$$
A_{i_{1}} \ldots i_{i n-1}=\frac{1}{(n-1) !} \sum_{p} \delta_{p} \prod_{k=1}^{n-1} \xi_{i_{p}(k)}^{(k)}
$$

Here, the sum runs over all permutations $p$ of the numbers $1, \ldots, n-1$ and

$$
\delta_{p}=\left\{\begin{array}{c}
1 \text { for even permutations of } 1, \ldots, n-1 \\
-1 \text { for odd permutations of } 1, \ldots, n-1
\end{array}\right.
$$


This construction has a meaning only when $n>2$. Because of the antisymmetry we can confine ourselves to index combinations $i_{1}<i_{2}<\ldots<i_{n-1}$. There are altogether $n$ possibilities of this type

$$
\begin{aligned}
& A^{k}=A_{1}, \ldots, k-1, k+1, \ldots, n \\
= & \frac{1}{(n-1) !}\left[\xi_{1}^{(1)} \ldots \xi_{k-1}^{(k-1)} \xi_{k+1}^{(k)} \ldots \xi_{n}^{(n-1)}-\ldots\right] .
\end{aligned}
$$

The $A^{k}$ span an $n$-dimensional vector space denoted

That this vector space is invariant is immediately clear and we proceed to prove the irreducibility. Every $A^{k}$ is an eigenvector of the diagonal generators. For the non-diagonal ones

$$
\begin{aligned}
& \mathbf{M}_{k l} A^{m}=A_{1}, \ldots, l-1, k, l+1, \ldots, m-1, m+1, \ldots, n \\
= & \delta_{m k}(-)^{m-l-1} A^{l}, \quad k \neq l .
\end{aligned}
$$

Defining

$$
\xi^{k}=(-1)^{k} A^{k}
$$

we have

$$
\begin{aligned}
& \mathbf{M}_{k l} \xi^{m}=-\delta_{k m} \xi^{l}, \quad k \neq l, \\
& \mathbf{M}_{k k} \xi^{m}=-\left(\delta_{k m}-\frac{1}{n}\right) \xi^{m} .
\end{aligned}
$$

By comparing (68) and (38) we can determine the connection between the $n \times n$ - mâtrix representing the element $a$ in the original space $\square$ and the $n \times n$ - matrix representing the same $a$ in the space $\left.\begin{array}{c}\square \\ \vdots\end{array}\right\}(n-1)$. To fix our conventions we make the correspondence $\xi_{k}<->\xi^{k}$ between the two vector spaces. In the space $\square$ the generators are represented by the $n \times n-$ matrices $M_{k l}$ of (34) and the element $a$ by $n \times n$ - matrix

$\left.\begin{array}{c}a=e^{i \sum \alpha_{k l} M_{k l}} . \\ \text { In the space } \quad \vdots \\ \square\end{array}\right\}(n-1)$ we have the same parameters $\alpha_{k l}$, but from (68) we see that the

generators are now represented by the $n \times n$ - matrices $M_{k l}=-M_{k l}$. The element $a$ is then represented by $a^{\prime}$,

$$
a^{\prime}=e^{i \sum \alpha_{k l}\left(-M_{k l}\right)}=\tilde{a}^{*} \text {. }
$$

Here we have used the property $\alpha_{k l}^{*}=\alpha_{l k}$. Thus, if an element $a \in S U(n)$ in $\square$ is 
represented by the matrix $a$ it is in $\left.\begin{array}{c}\square \\ \vdots \\ \square\end{array}\right\}(n-1)$ represented by the matrix $\tilde{a}^{*}$. It is also clear that the transformation $a \rightarrow \vec{a}^{*}$ preserves the algebraic properties of the elements of $S U(n)$ as $a_{1} a_{2} \rightarrow\left(\widetilde{a_{1} a_{2}}\right)^{*}=\widetilde{a}_{1}^{*} \tilde{a}_{2}^{*}$.

This latter description is particularly suitable for a discussion of the conjugate representation when $n=2$. For $a \in S U(2)$ the transformation $a \rightarrow \widetilde{a}^{*}$ is an equivalence transformation since

$$
\tilde{a}^{*}=\zeta a \zeta^{-1} \quad \zeta=\left(\begin{array}{cc}
0 & 1 \\
-1 & 0
\end{array}\right)
$$

Under this transformation the basis vectors are changed to

$$
\begin{aligned}
& \xi^{1}=\sum_{1}(\zeta)_{1 l} \xi_{l}=\xi_{2} . \\
& \xi^{2}=\sum_{l}(\zeta)_{2 l} \xi_{l}=-\xi_{1} .
\end{aligned}
$$

For $n=2$ the two representations are thus equivalent.

Let us return to the general $n$-dimensional case. Starting from our two fundamental n-dimensional vector spaces we can construct new representations by considering the space

$(n-1)\left\{\begin{array}{l}\square \\ \vdots \\ \square\end{array} \times \square\right.$ spanned by the basis vectors $\xi^{k} \xi_{l}$. We want to decompose this space into invariant and irreducible subspaces. To this end we make use of the identity

$$
\xi^{k} \xi_{l} \equiv \delta_{l}^{k} \frac{1}{n} \sum_{m=1}^{n} \xi^{m} \xi_{m}+\left[\xi^{k} \xi_{l}-\delta_{l}^{k} \frac{1}{n} \sum_{m=1}^{n} \xi^{m} \xi_{m}\right]
$$

where

$$
\delta_{l}^{k}= \begin{cases}1, & \text { if } k=l \\ 0, & \text { otherwise } .\end{cases}
$$

The space spanned by $\sum_{m=1}^{n} \xi^{m} \xi_{m}$ is one-dimensional, invariant and irreducible. This can
seen from

$$
\begin{aligned}
& \mathbf{M}_{k k} \sum \xi^{m} \xi_{m}=0, \\
& M_{k l} \sum \xi^{m} \xi_{m}=-\xi^{l} \xi_{k}+\xi^{l} \xi_{k}=0, \quad k \neq l .
\end{aligned}
$$


The space spanned by

$$
F_{l}^{k}=\xi^{k} \xi_{l}-\frac{1}{n} \delta_{l}^{k} \sum \xi^{m} \xi_{m}
$$

Is also invariant and irreducible. We first discuss the invariance. Every vector $F_{l}^{k}$ is an eigenvector of the diagonal operators $M_{\alpha \alpha}$. For the non-diagonal $M_{\alpha \beta}, \alpha \neq \beta$,

$$
M_{\alpha \beta} F_{l}^{k}=M_{\alpha \beta} \xi^{k} \xi_{l}=-\delta_{\alpha k} \xi^{\beta} \xi_{l}+\delta_{\beta l} \xi^{k} \xi_{\alpha}=-\delta_{\alpha k} F_{l}^{\beta}+\delta_{\beta l} F_{\alpha}^{k} .
$$

and so the invariance is proved.

We then turn to the irreducibility. We first prove that every basis vector can be transformed to $F_{2}^{1}$ and then that every basis vector can be obtained from $F_{2}^{1}$. If one index in $F_{l}^{k}$ is correct, e.g.

$$
M_{2 i} F_{i}^{1}=F_{2}^{1}, \quad i \neq 2
$$

If no index in $F_{l}^{k}$ is correct

$$
M_{2 l} M_{k 1} F_{l}^{k}=M_{2 l} M_{k 1} \xi^{k} \xi_{l}=-\left(1+\delta_{l l} \delta_{k 2}\right) F_{2}^{1} .
$$

Every non-diagonal $F_{l}^{k}$ is readily obtained from $F_{2}^{1}$. If no index is correct

$$
M_{j 2} M_{1 i} F_{2}^{1}=-\left(1+\delta_{i 2} \delta_{j 1}\right) F_{j}^{i} \text {. }
$$

and even simpler if one index is already correct. From the non-diagonal elements the diagonal ones are constructed by

$$
\begin{gathered}
\sum_{i \neq 1} M_{1 i} M_{i 2} F_{2}^{1}=\sum_{i \neq 1} M_{1 i} M_{i 2} \xi^{1} \xi_{2}=\sum_{i \neq 1} M_{1 i} \xi^{1} \xi_{i} \\
=-\sum_{i \neq 1} \xi^{i} \xi_{i}+\sum_{i \neq 1} \xi^{1} \xi_{1}=-\sum_{i \neq 1} \xi^{i} \xi_{i}+(n-1) \xi^{1} \xi_{1}=n F_{1}^{1} .
\end{gathered}
$$

e have now shown that the space $(n-1)\left\{\begin{array}{c}\square \\ \vdots \\ \square\end{array} \times \square\right.$ can be decomposed into two invariant and and irreducible subspaces and write

$$
(n-1)\left\{\begin{array}{l}
\square \\
\vdots \\
\square
\end{array} \times \square=(n)\left\{\begin{array}{l}
\square \\
\vdots \\
\square
\end{array}+(n-1)\left\{\begin{array}{l}
\square \\
\vdots \\
\square
\end{array}\right.\right.\right.
$$

he space $\left.\begin{array}{c}\square \\ \vdots \\ \square\end{array}\right\}(n)$ is spanned by $\sum_{m} \xi^{m} \xi_{m}$ and is 1-dimensional. As the original space is $n^{2}-$ Aimensional, the space $(n-1)\left\{\begin{array}{l}\square \\ \vdots \\ \square\end{array}\right.$ spanned by $F_{l}^{k}$ must be $\left(n^{2}-1\right)$-dimensional. This is also 
clear from the fact that we have one additional condition for the $F_{l}^{k}$ as $\sum_{k} F_{k}^{k}=0$ giving only $n^{2}-1$ linearly independent vectors.

We also note that $\left.\square \neq \begin{array}{c}\square \\ \vdots \\ \square\end{array}\right\}(n-1)$ for $n>2$. This is easily seen if we compare the decompositions (80) and (47) and note that $\frac{n(n-1)}{2}>1$ for $n>2$.

\section{Application to SU(3)}

The two fundamental 3-dimensional representations are $\square \equiv \underline{3}$ and $\quad \boxminus \equiv \underline{3}^{*}$. In $\underline{3}$ the basis is

$$
\xi_{1}=\left(\begin{array}{l}
1 \\
0 \\
0
\end{array}\right), \quad \xi_{2}=\left(\begin{array}{l}
0 \\
1 \\
0
\end{array}\right), \quad \xi_{3}=\left(\begin{array}{l}
0 \\
0 \\
1
\end{array}\right)
$$

Isotopic spin and hypercharge operators are introduced as

$$
\begin{aligned}
& T_{1}=\frac{1}{2}\left(M_{12}+M_{21}\right)=\frac{1}{2}\left(\begin{array}{lll}
0 & 1 & 0 \\
1 & 0 & 0 \\
0 & 0 & 0
\end{array}\right) . \\
& T_{2}=\frac{1}{2 i}\left(M_{12}-M_{21}\right)=\frac{1}{2 i}\left(\begin{array}{rrr}
0 & 1 & 0 \\
-1 & 0 & 0 \\
0 & 0 & 0
\end{array}\right) \text {. } \\
& T_{3}=\frac{1}{2}\left(M_{11}-M_{22}\right)=\frac{1}{2}\left(\begin{array}{rrr}
1 & 0 & 0 \\
0 & -1 & 0 \\
0 & 0 & 0
\end{array}\right) \text {. } \\
& \bar{T}^{2}=T_{1}^{2}+T_{2}^{2}+T_{3}^{2}=\left(\begin{array}{ccc}
3 / 4 & 0 & 0 \\
0 & 3 / 4 & 0 \\
0 & 0 & 0
\end{array}\right) . \\
& Y=-M_{33}=\frac{1}{3}\left(\begin{array}{rrr}
1 & 0 & 0 \\
0 & 1 & 0 \\
0 & 0 & -2
\end{array}\right) \text {. }
\end{aligned}
$$


it is easily checked that $\left[T_{1}, T_{2}\right]=i T_{3}$ cycl. and that $\left[T_{i}, Y\right]=0$. In the representation $\underline{3}^{*}$ he basis vectors are

$$
\begin{aligned}
& \xi^{1}=-\frac{1}{2}\left(\xi_{2} \eta_{3}-\xi_{3} \eta_{2}\right), \\
& \xi^{2}=\frac{1}{2}\left(\xi_{1} \eta_{3}-\xi_{3} \eta_{1}\right), \\
& \xi^{3}=-\frac{1}{2}\left(\xi_{1} \eta_{2}-\xi_{2} \eta_{1}\right) .
\end{aligned}
$$

the quantum numbers of these states are easily obtained as $Y$ is additive and $T$ adds according to angular momentum rules. We collect our results in a $Y T_{3}$-diagram.

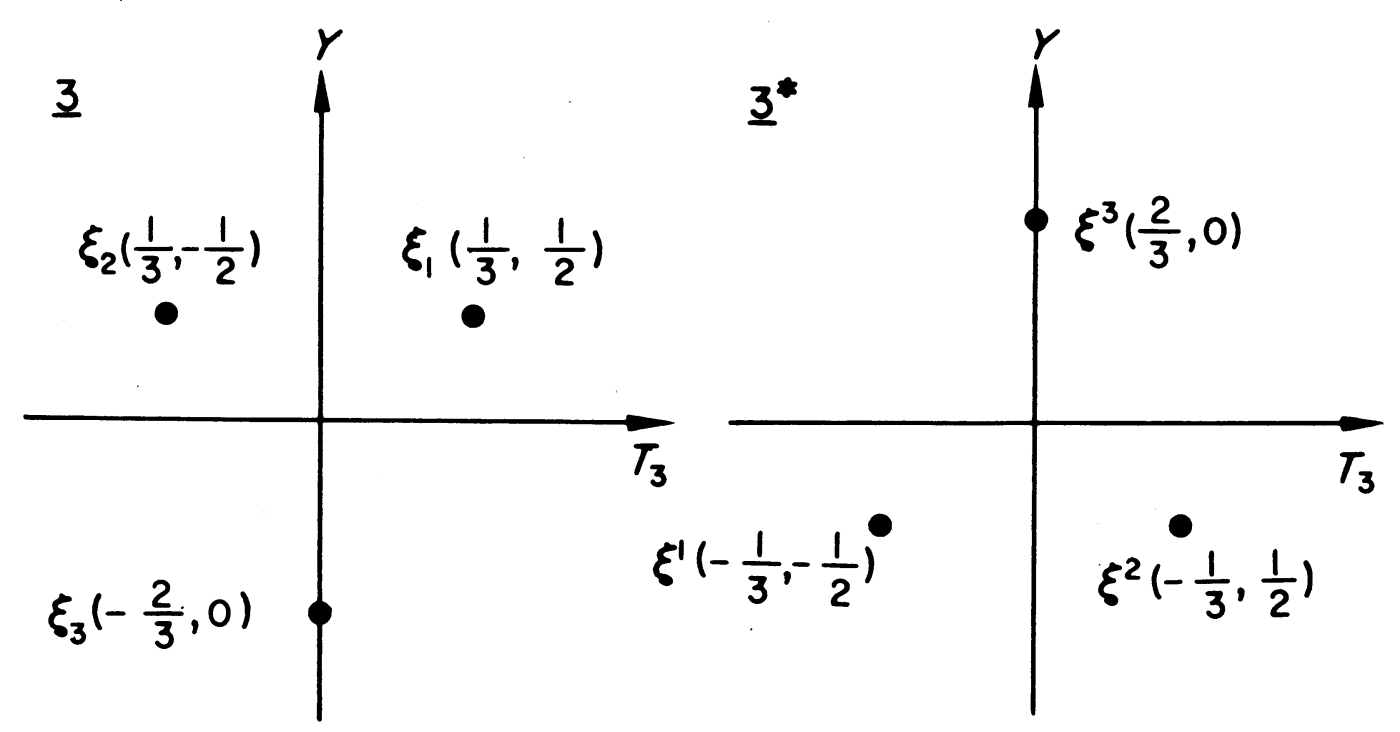

FIGURE 1

The states of the representations $\underline{3}$ and $\underline{3}^{*}$.

The charge is $Q=T_{3}+\frac{1}{2} Y=-M_{11}$. With this definition we find in $\underline{3}$ the values $\frac{2}{3},-\frac{1}{3}$, $\frac{1}{3}$, and in $3^{*}$ the values $-\frac{2}{3}, \frac{1}{3}, \frac{1}{3}$. When studying electromagnetic interactions it is more conenient to replace the classification according to isotopic spin by a classification according $U$-spin. The three components of $U$-spin are

$$
\begin{aligned}
& U_{1}=\frac{1}{2}\left(M_{23}+M_{32}\right)=\frac{1}{2}\left(\begin{array}{lll}
0 & 0 & 0 \\
0 & 0 & 1 \\
0 & 1 & 0
\end{array}\right) . \\
& U_{2}=\frac{1}{2 i}\left(M_{23}-M_{32}\right)=\frac{1}{2 i}\left(\begin{array}{rrr}
0 & 0 & 0 \\
0 & 0 & 1 \\
0 & -1 & 0
\end{array}\right) .
\end{aligned}
$$




$$
\begin{aligned}
& U_{3}=\frac{3}{4} Y-\frac{1}{2} T_{3}=\left(\begin{array}{ccc}
0 & 0 & 0 \\
0 & 1 / 2 & 0 \\
0 & 0 & -1 / 2
\end{array}\right) . \\
& \bar{U}^{2}=U_{1}^{2}+U_{2}^{2}+U_{3}^{2}=\left(\begin{array}{ccc}
0 & 0 & 0 \\
0 & 3 / 4 & 0 \\
0 & 0 & 3 / 4
\end{array}\right),
\end{aligned}
$$

The importance of $U$-spin can be traced back to

$$
\left[Q, U_{i}\right]=\left[-M_{11}, U_{i}\right]=0, \quad i=1,2,3 .
$$

From (85) we see that $\xi_{2}$ and $\xi_{3}$ with charge $-\frac{1}{3}$ have $U$-spin $\frac{1}{2}$ and that $\xi_{1}$ with charge $\frac{2}{3}$ has $U$-spin 0 .

Let us now classify particles according to representations of $S U(3)$. As a particle has integral charge we cannot use $\underline{3}$ or $\underline{3}^{*}$ but must go to higher dimensional representations. The basis vectors are then formal products of basis vectors in 3 . When the number of basis vectors in the product is a multiple of 3 we see that $Q$ is integral. Natural representations of physical interest are therefore

$$
\square \equiv \underline{10} \quad, \quad \forall \equiv \underline{8}, \quad \exists \equiv \underline{B} .
$$

The dimensions follow from the general results of Section 2. We now determine the isotopic spin content of these representations. By $(Y, T)$ we denote the $(2 T+1)$-dimensional space spanned by the states with total isotopic spin $T$ and hypercharge $Y$. Thus

$$
\begin{aligned}
& \underline{3}=\left(\frac{1}{3}, \frac{1}{2}\right)+\left(-\frac{2}{3}, 0\right) \\
& \underline{3}^{*}=\left(-\frac{1}{3}, \frac{1}{2}\right)+\left(\frac{2}{3}, 0\right),
\end{aligned}
$$

and

$$
\begin{aligned}
& \underline{3} \times \underline{3} \times \underline{3}=\underline{10}+2 \cdot \underline{3}+1=\left[\left(\frac{1}{3}, \frac{1}{2}\right)+\left(-\frac{2}{3}, 0\right)\right] \\
& \times\left[\left(\frac{1}{3}, \frac{1}{2}\right)+\left(-\frac{2}{3}, 0\right)\right] \times\left[\left(\frac{1}{3}, \frac{1}{2}\right)+\left(-\frac{2}{3}, 0\right)\right] .
\end{aligned}
$$

When evaluating the product on the right we note that $Y$ is additive and that $T$ adds according to angular momentum rules. The right-hand side can therefore be simplified using well-known rules from the theory of angular momentum, and as an example 


$$
\begin{aligned}
& \quad\left(\frac{1}{3}, \frac{1}{2}\right) \times\left(\frac{1}{3}, \frac{1}{2}\right) \times\left(-\frac{2}{3}, 0\right)=\left(\frac{1}{3}, \frac{1}{2}\right) \times\left(-\frac{1}{3}, \frac{1}{2}\right) \\
& =(0,1)+(0,0) .
\end{aligned}
$$

n this way we find

$$
\begin{aligned}
& \frac{10}{2}+2 \cdot \underline{8}+1 \equiv\left(1, \frac{3}{2}\right)+2\left(1, \frac{1}{2}\right)+3(0,1)+3(0,0) \\
& +3\left(-1, \frac{1}{2}\right)+(-2,0) .
\end{aligned}
$$

s 8 appears twice on the left it can contain only $\left(1, \frac{1}{2}\right),(0,1),(0,0),\left(-1, \frac{1}{2}\right)$ and each of hese at most once. But they give together 8 states. This yields

$$
\underline{8}=\left(1, \frac{1}{2}\right)+(0,1)+(0,0)+\left(-1, \frac{1}{2}\right) \text {. }
$$

rom our previous discussion it follows that $\underline{1}=(0,0)$ and, consequently,

$$
10=\left(1, \frac{3}{2}\right)+(0,1)+\left(-1, \frac{1}{2}\right)+(-2,0) \text {. }
$$

From (84) it is clear that the vectors of $\underline{3}^{*} \times \underline{3}$ have integral values for the charge. From ur reduction formula (80)

$$
\underline{3} \times \underline{3}^{*}=\underline{1}+\underline{8} \text {. }
$$

here $\underline{1}=(0,0)$. As

$$
\begin{aligned}
& \underline{3} \times \underline{3}^{*}=\left[\left(\frac{1}{3}, \frac{1}{2}\right)+\left(-\frac{2}{3}, 0\right)\right] \times\left[\left(-\frac{1}{3}, \frac{1}{2}\right)+\left(\frac{2}{3}, 0\right)\right] \\
&=\left(1, \frac{1}{2}\right)+2(0,0)+(0,1)+\left(-1, \frac{1}{2}\right) .
\end{aligned}
$$

find

$$
\underline{8}=\left(1, \frac{1}{2}\right)+(0,0)+(0,1)+\left(-1, \frac{1}{2}\right) .
$$

Just as for $\underline{3}_{\text {and }} \underline{3}^{*}$ we can visualize the states and now also the particles corresponding to 8 and 10 in a $Y T_{3}$-diagram (Fig. 2). We note that the state $T=Y=0$ in the vector meson bctet is a mixture of $\omega$ and $\varphi$. 

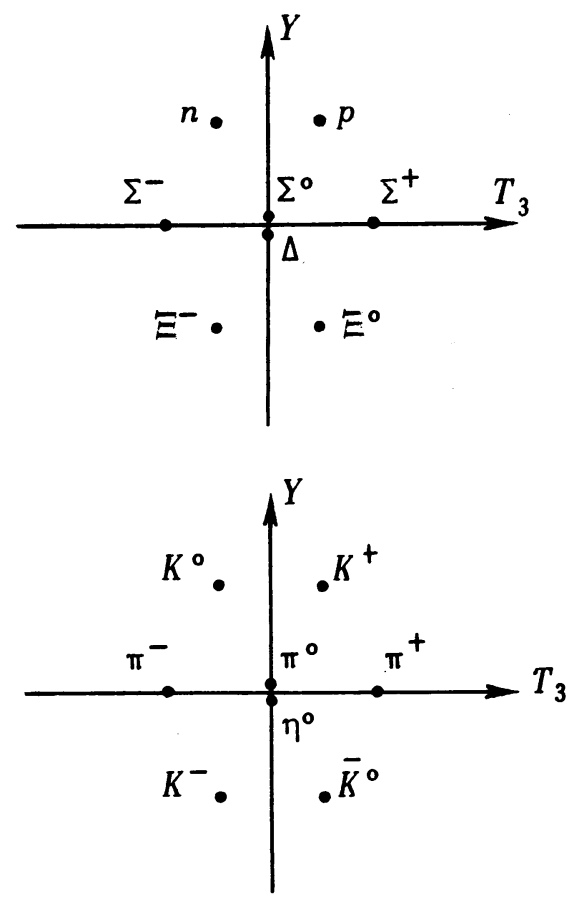
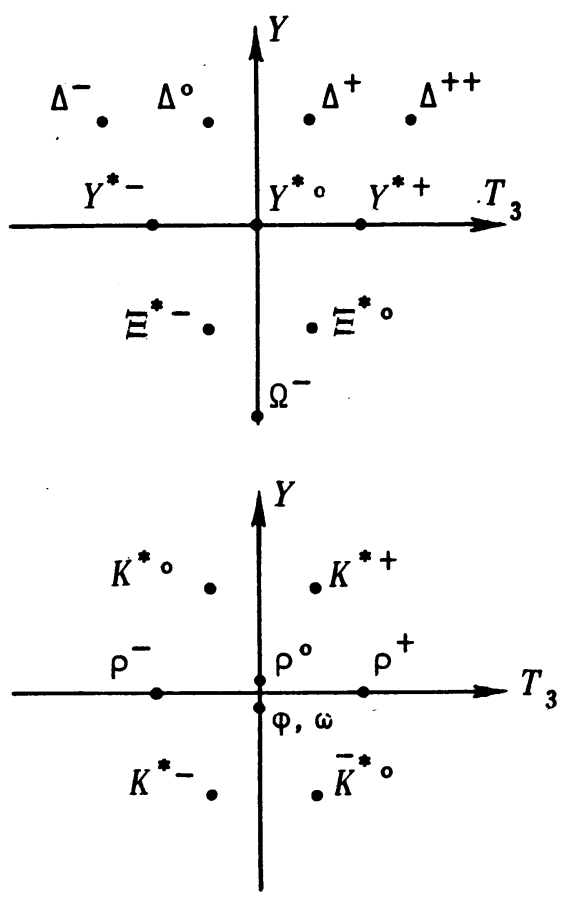

FIGURE 2

These figures display the states of (a) the baryon octet; (b) the baryon decuplet; (c) the scalar vector octet; (d) the vector meson octet.

\section{Application to SU(6)}

The group $S U(6)$ is used to include also a non-relativistic description of the spin of the particles. Let us see how this is done. The generators $M_{k l}$ of the fundamental 6-dimensional representation $\square \equiv \underline{6}$ are $6 \times 6$ matrices defined by (35). The group $S U(6)$ contains the two commuting subgroups $S \bar{U}(3)$ and $S U(2)$.

The operators of the $S U(2)$ subgroup can be chosen as

$$
\begin{aligned}
& M_{12}^{(2)}=\sum_{i=1}^{3} M_{i, i+3}=\left(\begin{array}{ll}
0 & 1 \\
0 & 0
\end{array}\right), \\
& M_{21}^{(2)}=\sum_{i=1}^{3} M_{i+3, i}=\left(\begin{array}{ll}
0 & 0 \\
1 & 0
\end{array}\right) . \\
& M_{11}^{(2)}=-M_{22}^{(2)}=\frac{1}{2} \sum_{i=1}^{3}\left(M_{i i}-M_{i+3, i+3}\right)=\frac{1}{2}\left(\begin{array}{cc}
1 & 0 \\
0 & -1
\end{array}\right) .
\end{aligned}
$$


very submatrix in the $2 \times 2$ matrices on the right is a $3 \times 3$ matrix and 1 stands for the unit $1 \times 3$ matrix. If the operators of this $S U(2)$ subgroup are interpreted as spin operators, the if basis vectors in $S U(6)$ have spin $\frac{1}{2}$ and $\xi_{i}, i=1,2,3$, have $S_{3}=\frac{1}{2}$ while $\xi_{i}, i=4,5,6$, lave $S_{3}=-\frac{1}{2}$.

The operators of the $S U(3)$ subgroup are

$$
M_{k l}^{(3)}=M_{k l}+M_{l+3, k+3}=\left(\begin{array}{cc}
M_{k l} & 0 \\
& \\
0 & M_{k l}
\end{array}\right), k, l=1,2,3,
$$

here the submatrices on the right are the fundamental $3 \times 3$ matrices of $S U(3)$ defined by (35). therefore, the states $\xi_{i}, i=1,2,3$, and $\xi_{i}, i=4,5,6$, each belong to a fundamental riplet 3 .

(3)

Every operator of the group $S U(2)$ commutes with every operator of the group $S U(3)$. Thereore, $S U(3) \times S U(2)$ is a subgroup of $S U(6)$ and every basis vector of $S U(6)$ is a simultaneas eigenvector of $S U(2)-$ and $S U(3)$ - operators. We find, e.g. that $\xi_{1}$ has $=\frac{1}{3}, T_{3}=\frac{1}{2}$ and $S_{3}=\frac{1}{2}$.

As $S U(3) \times S U(2)$ is a subgroup of $S U(6)$ we can write $\square \equiv \underline{6}=(\underline{3}, \underline{2})$ where in the ranthesis we have used the dimensions of the $S U(3)$ - and $S U(2)$-groups. From the transprmation properties derived in Section 3

$$
\text { 日 } \equiv \underline{6}^{*}=\left(\underline{3}^{*}: \underline{2}^{*}\right)=\left(\underline{3}^{*}, \underline{2}\right) \text {. }
$$

howing the $S U(3) \times S U(2)$ content of these two fundamental representations we proceed to termine the decompositions for the other representations of physical interest.

We then note that the values for $Q=T_{3}+\frac{1}{2} Y$ in $\underline{6}$ are the same as in the $I S U(3)$ representaion 3 . Therefore, the number of products of basic representations in a physical representation ust be a multiple of 3 . Natural candidates are

$$
\square \equiv \underline{56}, \quad \square \equiv \underline{70}, \quad \text { G } \equiv \underline{20} \text {. }
$$

dimensions are obtained from the general results of Section 2 . We use the formula (64)

$$
\underline{6} \times \underline{6} \times \underline{6}=\underline{56}+2 \cdot \underline{70}+\underline{20}
$$

determine the $S U(3) \times S U(2)$ content of these representations. We have

$$
\begin{gathered}
\underline{6} \times \underline{6} \times \underline{6}=(\underline{3}, \underline{2}) \times(\underline{3}, \underline{2}) \times(\underline{3}, \underline{2}) \\
=(\underline{3} \times \underline{3} \times \underline{3}, \underline{2} \times \underline{2} \times \underline{2})=(\underline{10}+2 \cdot \underline{8}+\underline{1}, \underline{4}+2 \cdot \underline{2}) \\
=(\underline{10}, \underline{4})+2(\underline{10}, \underline{2})+2(\underline{8}, \underline{4})+4(\underline{8}, \underline{2})+(\underline{1}, \underline{4})+2(\underline{1}, \underline{2}) .
\end{gathered}
$$


The $S U(3)$ - part is reduced by $(90)$. As $(10, \underline{4})$ contains 40 states and appears only once it mu belong to $\underline{56}$. In this representation we need 16 more states. The only possibility is $(\underline{8}, \underline{2})$ and thus

$$
\underline{56}=(\underline{10}, \underline{4})+(\underline{8}, \underline{2}) .
$$

In the same way there is only one way to fill 20

$$
\underline{20}=(\underline{8}, \underline{2})+(\underline{1}, \underline{4})
$$

and consequently

$$
\underline{70}=(\underline{10}, \underline{2})+(\underline{8}, \underline{4})+(\underline{8}, \underline{2})+(\underline{1}, \underline{2}) .
$$

The space $\underline{6}^{*} \times \underline{6}$ has basis vectors that are formal products of 6 basis vectors belonging to different representations $\underline{6}$. Therefore, the vectors of $\underline{6}^{*} \times \underline{6}$ have integral values for the charge and $\underline{6}^{*} \times \underline{6}$ is a physically acceptable space. From (80)

$$
\underline{6}^{*} \times \underline{6}=\underline{1}+\underline{35} \text {. }
$$

This reduction produces one new interesting representation, $\underline{35}$. We determine its $S U(3) \times S U$ content

$$
\begin{aligned}
& \underline{6}^{*} \times \underline{6}=\left(\underline{3}^{*}, \underline{2}\right) \times(\underline{3}, \underline{2})=\left(\underline{3}^{*} \times \underline{3}, \underline{2} \times \underline{2}\right)=(\underline{1}+\underline{8}, \underline{1}+\underline{3}) \\
= & (\underline{1}, \underline{1})+(\underline{1}, \underline{3})+(\underline{8}, \underline{1})+(\underline{8}, \underline{3}),
\end{aligned}
$$

where the reduction is performed by means of (95). Thus

$$
\underline{35}=(\underline{8}, \underline{1})+(\underline{8}, \underline{3})+(\underline{1}, \underline{3})
$$

is a possible physical representation.

Particles are grouped into representations in the following way. In 56 we put the baryon octet containing particles $(n, p, \Sigma, \Lambda)$ with spin $\frac{1}{2}$ and the baryon decuplet containing particles $\left(\Delta, Y^{*}, \Xi^{*}, \Omega^{-}\right)$with spin $\frac{3}{2}$. In 35 we put the mesons. The $(\underline{8}, \underline{1})$ is filled by the pseudoscalar mesons $(\pi, \eta, K)$. For the vector mesons a complication appears as the physical particles $\phi$ and $\omega$ are both mixtures of the states $Y=T=0$ in $(\underline{8} . \underline{3})$ and $(\underline{1}, \underline{3})$. This mixing c. be explained by further physical assumptions. The other states in $(\underline{8}, \underline{3})$ are occupied by $\rho$ and $K^{*}$. No other $S U(6)$ - representation can be filled by the presently known particles.

\section{Acknowledgment}

I would like to thank Professor Gunnar Källén for very helpful discussions on this subject The note was prepared for a seminar directed by him. 
1.3, No. 1

\section{References}

Y. NE' EMAN, Nucl. Phys. 26, 222 (1961); M. GELL-MANN, California Institute of Technology Report CTSL-20 (unpublished); also reprinted in, M. GELL-MANN and Y. NE' EMAN, The Eightfold Way. Benjamin, New York (1964).

D. L. PURSEY, Proc. Roy. Soc. A275, 284 (1963).

F. GÜRSEY and L.A. RADICATI, Phys. Rev. Letters 13, 173 (1964); A. PAIS, Phys. Rev. Let ters 13, 175 (1964).

S. OKUBO, Phys. Letters 5, 165 (1963); M. A. B. BEG and V. SINGH, Phys. Rev. Letters, 13, 418 (1964); T.K. KUO and T. YAO, Phys. Rev. Letters 13, 415 (1964).

H. BOERNER, Representations of Groups. North Holland, Amsterdam (1963).

A.S. WIGHTMAN, L'invariance dans la mécanique quantique relativiste, in Relations de dispersion et Particules Elementaires. Hermann, Paris (1960). 